PROCEEDINGS OF THE

AMERICAN MATHEMATICAL SOCIETY

Volume 134, Number 5, Pages 1391-1396

S 0002-9939(05)08084-6

Article electronically published on October 7, 2005

\title{
AN IMAGE PROBLEM FOR COMPACT OPERATORS
}

\author{
ISABELLE CHALENDAR AND JONATHAN R. PARTINGTON
}

(Communicated by Joseph A. Ball)

\begin{abstract}
Let $\mathcal{X}$ be a separable Banach space and $\left(\mathcal{X}_{n}\right)_{n}$ a sequence of closed subspaces of $\mathcal{X}$ satisfying $\mathcal{X}_{n} \subset \mathcal{X}_{n+1}$ for all $n$. We first prove the existence of a dense-range and injective compact operator $K$ such that each $K \mathcal{X}_{n}$ is a dense subset of $\mathcal{X}_{n}$, solving a problem of Yahaghi (2004). Our second main result concerns isomorphic and dense-range injective compact mappings between dense sets of linearly independent vectors, extending a result of Grivaux (2003).
\end{abstract}

\section{INTRODUCTION}

Let $\mathcal{X}$ be an infinite-dimensional separable real or complex Banach space and denote by $\mathcal{L}(\mathcal{X})$ the algebra of all bounded and linear mappings from $\mathcal{X}$ to $\mathcal{X}$. $\mathrm{A}$ chain of subspaces of $\mathcal{X}$ is defined to be a sequence (at most countable) $\left(\mathcal{X}_{n}\right)_{n \geq 0}$ of closed subspaces of $\mathcal{X}$ such that $\mathcal{X}_{0}=\{0\}$ and $\mathcal{X}_{n} \subset \mathcal{X}_{n+1}$ for all $n \geq 0$. The identity map on $\mathcal{X}$ is denoted by $\mathrm{Id}$.

Section 2 of this paper is devoted to the construction of injective and denserange compact operators $K \in \mathcal{L}(\mathcal{X})$ such that $K \mathcal{X}_{n}$ is a dense subset of $\mathcal{X}_{n}$ for all $n$. Note that for separable Hilbert spaces, using an orthonormal basis $\mathcal{B}$ and diagonal compact operators relative to $\mathcal{B}$, it is not difficult to construct injective, dense-range and normal compact operators such that each $K \mathcal{X}_{n}$ is a dense subset of $\mathcal{X}_{n}$. Nevertheless, in the case of an arbitrary Banach space, the construction is not so obvious. In [6], Yahaghi obtained partial results. He proved that there exist dense-range compact operators $K$ such that $K \mathcal{X}_{n}$ is a dense subset of $\mathcal{X}_{n}$ and there exist injective compact operators such that $K \mathcal{X}_{n} \subset \mathcal{X}_{n}$.

Section 3 of the paper was motivated by Lemma 2.1 of [1], asserting that, on a general separable Banach space $\mathcal{X}$, two dense sets of linearly independent vectors of $\mathcal{X}$ are isomorphic. We construct injective, dense-range and compact mappings between dense sets of linearly independent vectors in $\mathcal{X}$.

In our construction we shall make use of biorthogonal sequences in Banach spaces. Recall that a sequence $\left(x_{n}, x_{n}^{*}\right)$ in $\mathcal{X}$, where each $x_{n}$ lies in $\mathcal{X}$ and $x_{n}^{*}$ in $\mathcal{X}^{*}$, is biorthogonal if $x_{n}^{*}\left(x_{m}\right)=0$ for $m \neq n$ while $x_{n}^{*}\left(x_{n}\right)=1$ for all $n$. It is fundamental if the linear span of the vectors $x_{n}$ is dense, and it is total if the only vector $x$ such that $x_{n}^{*}(x)=0$ for all $n$ is the zero vector. Every separable Banach space possesses fundamental total biorthogonal sequences, and they can even be

Received by the editors November 3, 2004 and, in revised form, December 9, 2004.

2000 Mathematics Subject Classification. Primary 47A15, 47A46, $47 \mathrm{~B} 07$.

Key words and phrases. Chains of invariant subspaces, compact operators, Banach spaces.

(C)2005 American Mathematical Society Reverts to public domain 28 years from publication 
found such that $\left(\left\|x_{n}\right\|\left\|x_{n}^{*}\right\|\right)$ is bounded [3]. We refer to [2] for a detailed account of this subject.

\section{InJeCTIVE AND DENSE-RANGE COMPACT OPERATORS PRESERVING CHAINS}

Let $\left(\mathcal{X}_{n}\right)_{n \geq 0}$ be a chain of subspaces of $\mathcal{X}$. Our aim is to construct an injective and dense-range compact operator $K$ such that $K \mathcal{X}_{n}$ is a dense subset of $\mathcal{X}_{n}$ for all $n \geq 0$. The first result is elementary, but is the key to more elaborate constructions.

Proposition 2.1. Let $\mathcal{X}$ be a separable Banach space. Then there exists a compact operator on $\mathcal{X}$ that is injective and has dense range.

Proof. There exists a fundamental and total biorthogonal sequence $\left(x_{n}, x_{n}^{*}\right)_{n \geq 1}$ for $\mathcal{X}$ with $\left\|x_{n}\right\|=1$ for each $n$ (see [2]). We may assume if we like that the $\left(x_{n}^{*}\right)$ are also uniformly bounded, although this is not needed here. Define the compact operator $K$ by

$$
K x=\sum_{n=1}^{\infty} \frac{1}{n^{2}} \frac{x_{n}^{*}(x) x_{n}}{\left\|x_{n}^{*}\right\|} .
$$

Clearly the range of $K$ contains all finite linear combinations of the vectors $x_{n}$ and is dense since the $\left(x_{n}\right)$ are a fundamental system. If $K x=0$, then $x_{k}^{*}(K x)=0$ for each $k$, which implies that $x_{k}^{*}(x)=0$ for each $k$ and hence $x=0$ since the $\left(x_{n}^{*}\right)$ are total.

The following theorem gives a positive answer to a question posed by Yahaghi [6. p. 1063].

Theorem 2.1. Let $\mathcal{X}$ be an infinite-dimensional separable Banach space and $\mathcal{Y}$ a closed subspace of $\mathcal{X}$. Then there is a dense-range and injective compact operator $K$ such that $K \mathcal{Y}$ is a dense subspace of $\mathcal{Y}$.

Proof. Take fundamental total biorthogonal sequences $\left(y_{n}, y_{n}^{*}\right)$ and $\left(w_{n}, w_{n}^{*}\right)$ in the separable Banach spaces $\mathcal{Y}$ and $\mathcal{X} / \mathcal{Y}$ respectively. Using the Hahn-Banach theorem we may suppose that $y_{n}^{*}$ is extended to an element of $\mathcal{X}^{*}$, and we keep the same notation.

Note that the quotient mapping $p: \mathcal{X} \rightarrow \mathcal{X} / \mathcal{Y}$ is a contraction, and its adjoint is a mapping $p^{*}:(\mathcal{X} / \mathcal{Y})^{*} \rightarrow \mathcal{X}^{*}$. Define $x_{n}^{*}=p^{*}\left(w_{n}^{*}\right)$ for each $n$.

Let us suppose that both $\mathcal{Y}$ and $\mathcal{X} / \mathcal{Y}$ are infinite-dimensional: if one is finitedimensional the same construction works, with some of the sums being finite. Without loss of generality $\left\|y_{n}\right\|=1$ and $\left\|w_{n}\right\|=1$ for all $n$, so we can choose $x_{n} \in \mathcal{X}$ with $p\left(x_{n}\right)=w_{n}$ and $\left\|x_{n}\right\| \leq 2$.

Define

$$
K x=\sum_{n=1}^{\infty} \frac{1}{n^{2}} \frac{y_{n}^{*}(x) y_{n}}{\left\|y_{n}^{*}\right\|}+\sum_{n=1}^{\infty} \frac{1}{n^{2}} \frac{x_{n}^{*}(x) x_{n}}{\left\|x_{n}^{*}\right\|} .
$$

Clearly $K$ is a compact operator and, if $y \in \mathcal{Y}$, then $x_{n}^{*}(y)=\left(p^{*} w_{n}^{*}\right)(y)=w_{n}^{*}(p y)=$ 0 for each $n$, and so $K$ maps $\mathcal{Y}$ into $\mathcal{Y}$. Also the image space $K \mathcal{Y}$ is dense in $\mathcal{Y}$ as in Proposition 2.1 
Note that $K y_{n}$ is a nonzero multiple of $y_{n}$ and that $K x_{n}$ is a vector in $\mathcal{Y}$ plus a nonzero multiple of $x_{n}$, since

$$
x_{m}^{*}\left(x_{n}\right)=w_{m}^{*}\left(p x_{n}\right)=w_{m}^{*}\left(w_{n}\right)= \begin{cases}0 & \text { if } m \neq n, \\ 1 & \text { if } m=n .\end{cases}
$$

It remains to show that $K \mathcal{X}$ is dense in $\mathcal{X}$ and that $K$ is injective. If $x \in \mathcal{X}$, then for each $\varepsilon>0$ we can find a finite linear combination $w=\sum c_{n} w_{n}=p\left(\sum c_{n} x_{n}\right)$ with $\|p(x)-w\|<\varepsilon / 3$; thus there is a vector $y \in \mathcal{Y}$ with $\left\|x-\sum c_{n} x_{n}-y\right\|<2 \varepsilon / 3$, and finally a finite sum $\sum b_{n} y_{n}$ with $\left\|y-\sum b_{n} y_{n}\right\|<\varepsilon / 3$, giving

$$
\left\|x-\left(\sum c_{n} x_{n}+\sum b_{n} y_{n}\right)\right\|<\varepsilon .
$$

Now $\sum c_{n} x_{n}+\sum b_{n} y_{n}-K\left(\sum n^{2}\left\|x_{n}^{*}\right\| c_{n} x_{n}\right)$ lies in $\mathcal{Y}$, and $K \mathcal{Y}$ is dense in $\mathcal{Y}$, and we deduce that $K$ has dense range.

Suppose finally that $K x=0$. Applying $x_{m}^{*}$ to $K x$ we deduce that $x_{m}^{*}(x)=0$ for each $m$, and so $x \in \mathcal{Y}$. Hence

$$
K x=\sum_{n=1}^{\infty} \frac{1}{n^{2}} \frac{y_{n}^{*}(x) y_{n}}{\left\|y_{n}^{*}\right\|}=0 .
$$

Applying $y_{m}^{*}$ we deduce that $y_{m}^{*}(x)=0$ for each $m$ and hence $x=0$.

The above proof could be shortened, but would become less elementary, by applying stronger results on the extension of biorthogonal sequences. We shall use the above proof technique, together with the more difficult results, in order to deal with chains of subspaces, as in the next theorem; this also provides stronger forms of results given by Yahaghi [6].

Theorem 2.2. Let $\mathcal{X}$ be a separable infinite-dimensional Banach space and let $\left(\mathcal{X}_{n}\right)_{n \geq 0}$ be a chain of subspaces of $\mathcal{X}$. Then there is a dense range and injective compact operator $K$ such that $K \mathcal{X}_{n}$ is a dense subspace of $\mathcal{X}_{n}$ for all $n$.

Proof. We shall use the known result that a uniformly bounded fundamental total biorthogonal sequence for a subspace of a Banach space can be extended to a uniformly bounded fundamental total biorthogonal sequence for the larger space (cf. [4, 5]). Assuming this result, we construct the operator $K$ given by

$$
K x=\sum_{n=1}^{\infty} \frac{1}{n^{2}} \sum_{k=1}^{\infty} \frac{1}{k^{2}} \frac{x_{n k}^{*}(x) x_{n k}}{\left\|x_{n k}\right\|},
$$

where $\left\{\left(x_{j k}, x_{j k \mid \mathcal{X}_{n}}^{*}\right): j \leq n, k \geq 1\right\}$ is a uniformly bounded fundamental total biorthogonal sequence in each $\mathcal{X}_{n}$. (Our notation assumes that each $\mathcal{X}_{n+1} / \mathcal{X}_{n}$ is infinite-dimensional; an obvious modification should be made if not.)

If the union of the $\mathcal{X}_{n}$ is dense, then this is the operator required, as it can be shown in a fashion similar to Proposition 2.1 that this operator is compact and injective, mapping $\mathcal{X}_{n}$ densely into $\mathcal{X}_{n}$. Otherwise, let $\mathcal{Y}$ denote the closure of the union of the spaces $\mathcal{X}_{n}$. As in the proof of Theorem 2.1, we may find a biorthogonal sequence $\left(w_{n}, w_{n}^{*}\right)$ for $\mathcal{X} / \mathcal{Y}$ with $\left\|w_{n}\right\|=1$ for all $n$. Writing $p$ for the quotient mapping from $\mathcal{X}$ onto $\mathcal{X} / \mathcal{Y}$, we choose $x_{n} \in \mathcal{X}$ with $p\left(x_{n}\right)=w_{n}$ and $\left\|x_{n}\right\| \leq 2$ and 
let $x_{n}^{*}=p^{*}\left(w_{n}^{*}\right) \in \mathcal{X}^{*}$. The desired operator is given by

$$
K^{\prime} x=K x+\sum_{n=1}^{\infty} \frac{1}{n^{2}} \frac{x_{n}^{*}(x) x_{n}}{\left\|x_{n}^{*}\right\|}
$$

and its properties are verified as in the proof of Theorem 2.1.

\section{ISOMORPHIC AND COMPACT MAPPINGS BETWEEN DENSE INDEPENDENT SETS}

We first recall an ingenious lemma of S. Grivaux, which motivates what follows.

Lemma 3.1 (Lemma 2.1 in [1]). Let $V=\left\{v_{n}: n \geq 1\right\}$ and $W=\left\{w_{n}: n \geq 1\right\}$ be dense linearly independent sets in a Banach space $\mathcal{X}$. For any $\varepsilon>0$, there exists an isomorphism $L$ of $\mathcal{X}$ with $\|\mathrm{Id}-L\|<\varepsilon$ such that $L(V)=W$.

A simple way to construct a countable dense linearly independent set in a separable infinite-dimensional Banach space $\mathcal{X}$ is to begin by choosing a linearly independent set $U=\left\{u_{n}: n \geq 1\right\}$ of normalized vectors in $\mathcal{X}$ such that the linear span of $U$ is dense. Next, let $T=\left\{t_{m}: m \geq 1\right\}$ be an enumeration of the set of finite linear combinations of vectors in $U$ with coefficients in $\mathbb{Q}($ or $\mathbb{Q}+i \mathbb{Q}$ if the Banach space is complex). Then $T$ is dense, and hence so is the set $S=\left\{t_{m}+u_{f(m)} / m: m \geq 1\right\}$, for any $f: \mathbb{N} \rightarrow \mathbb{N}$. We can guarantee that $S$ is an independent set by choosing the sequence $(f(m))$ to be increasing sufficiently rapidly.

In order to obtain an analogous version of Lemma 3.1 replacing isomorphism by injective and dense-range compact operator, we first need the following observation.

Proposition 3.1. If $\left(v_{n}\right)$ is a dense independent set in $\mathcal{X}$ and $T: \mathcal{X} \rightarrow \mathcal{X}$ is a bounded linear operator that is injective with dense range, then $\left(T v_{n}\right)$ is also a dense independent set in $\mathcal{X}$.

Proof. Take any $x \in \mathcal{X}$ and choose vectors $y_{k}=T x_{k} \in T \mathcal{X}$ such that $\left\|y_{k}-x\right\| \rightarrow 0$. Choose $v_{n_{k}}$ such that $\left\|v_{n_{k}}-x_{k}\right\| \rightarrow 0$. Then

$$
\left\|T v_{n_{k}}-x\right\| \leq\|T\|\left\|v_{n_{k}}-x_{k}\right\|+\left\|y_{k}-x\right\| \rightarrow 0 .
$$

Thus the $\left(T v_{n}\right)$ form a dense set. Also, if $\sum_{n=1}^{N} a_{n} T v_{n}=0$, then $\sum_{n=1}^{N} a_{n} v_{n}=$ 0 , since $T$ is injective, so $a_{1}=a_{2}=\ldots=a_{N}=0$, and hence the $\left(T v_{n}\right)$ are independent.

Combining these results, we obtain the following.

Corollary 3.1. Let $V=\left\{v_{n}: n \geq 1\right\}$ and $W=\left\{w_{n}: n \geq 1\right\}$ be dense linearly independent sets in a separable Banach space $\mathcal{X}$. Then there is an injective compact operator $K$ (necessarily with dense range) such that $K(V)=W$.

Proof. Let $T$ be any compact injective operator on $\mathcal{X}$ with dense range, for example the one given in Proposition 2.1. By Proposition 3.1, the vectors $\left(T v_{n}\right)$ form a dense independent set $U$ in $\mathcal{X}$. Now by Lemma 3.1 there is an isomorphism $L$ such that $L(U)=W$. In other words, if we define $K=L T$, then $K$ is compact and injective with dense range, and $K(V)=L T(V)=L(U)=W$, as required.

In the spirit of the results of Section 2, we now look at mappings involving subspaces. The main result of this section is the following theorem. 
Theorem 3.1. Let $\mathcal{Y}$ be a closed subspace of a separable infinite-dimensional Banach space $\mathcal{X}$, and let $V_{1}, W_{1}$ be countable, dense, linearly independent subsets of $\mathcal{Y}$. Let $V_{2}, W_{2}$ be countable subsets of $\mathcal{X}$ that are linearly independent over $\mathcal{Y}$ (i.e., the sets of quotients $p\left(V_{2}\right)$ and $p\left(W_{2}\right)$ are independent in $\left.\mathcal{X} / \mathcal{Y}\right)$, such that $V_{1} \cup V_{2}$ and $W_{1} \cup W_{2}$ are dense (independent) subsets of $\mathcal{X}$. Then there is an invertible operator $L$ mapping $V_{1}$ onto $W_{1}$ and $V_{2}$ onto $W_{2}$; there is also a compact, injective, dense-range operator $K$ with the same mapping properties.

Proof. For the construction of $L$ we modify the argument of Grivaux in [1]. Let $V_{1}=$ $\left\{v_{1}, v_{2}, \ldots\right\}, W_{1}=\left\{w_{1}, w_{2}, \ldots\right\}$ and $V_{2}=\left\{t_{1}, t_{2}, \ldots\right\}$ and $W_{2}=\left\{u_{1}, u_{2}, \ldots\right\}$. We construct a convergent sequence of invertible operators on $\mathcal{X}$ that all map $\mathcal{Y}$ onto $\mathcal{Y}$, beginning with $L_{0}=\mathrm{Id}$. The sequence continues $I_{1}, J_{1}, K_{1}, L_{1}, I_{2}, J_{2}, K_{2}, L_{2}, I_{3}, \ldots$. If at any stage an operator already satisfies the additional mapping conditions required of its successor, then its successor is defined to be the same operator once more; otherwise the new operator is defined as a small perturbation of its predecessor (for example, with $\left\|T_{k+1}-T_{k}\right\| \leq 2^{-k}$, where $T_{k}$ denotes the $k$ th operator constructed).

We proceed as follows:

(1) Let $I_{1}=L_{0}+x_{1}^{*} \otimes x_{1}$ be such that $I_{1} v_{1}=w_{p_{1}}$ for some $p_{1}$; to achieve this choose $x_{1}^{*}$ with $x_{1}^{*}\left(v_{1}\right)=1$ and take $x_{1} \in \mathcal{Y}$ small such that $v_{1}+x_{1}=w_{p_{1}}$.

(2) Let $J_{1}=\left(I_{1}^{-1}+y_{1}^{*} \otimes y_{1}\right)^{-1}$ be such that $J_{1} v_{1}=w_{p_{1}}$ and $J_{1} v_{q_{1}}=w_{1}$ for some $q_{1}$; to achieve this choose $y_{1}^{*}$ with $y_{1}^{*}\left(w_{p_{1}}\right)=0, y_{1}^{*}\left(w_{1}\right)=1$, and take $y_{1} \in \mathcal{Y}$ small such that $I_{1}^{-1} w_{1}+y_{1}=v_{q_{1}}$.

(3) Let $K_{1}=J_{1}+z_{1}^{*} \otimes z_{1}$ be such that $K_{1} v_{1}=w_{p_{1}}, K_{1} v_{q_{1}}=w_{1}$, and $K_{1} t_{1}=u_{r_{1}}$ for some $r_{1}$; to achieve this choose $z_{1}^{*}$ such that $z_{1}^{*}(y)=0$ for all $y \in \mathcal{Y}$, $z_{1}^{*}\left(t_{1}\right)=1$, and take $z_{1} \in \mathcal{X}$ small such that $J_{1} t_{1}+z_{1}=u_{r_{1}}$.

(4) Let $L_{1}=\left(K_{1}^{-1}+a_{1}^{*} \otimes a_{1}\right)^{-1}$ be such that $L_{1} v_{1}=w_{p_{1}}, L_{1} v_{q_{1}}=w_{1}$, $L_{1} t_{1}=u_{r_{1}}$, and $L_{1} t_{s_{1}}=u_{1}$ for some $s_{1}$; to achieve this choose $a_{1}^{*}$ with $a_{1}^{*}(y)=0$ for all $y \in \mathcal{Y}, a_{1}^{*}\left(u_{r_{1}}\right)=0, a_{1}^{*}\left(u_{1}\right)=1$, and take $a_{1} \in \mathcal{X}$ small such that $K_{1}^{-1} u_{1}+a_{1}=t_{s_{1}}$.

We then cycle around through $I_{2}, J_{2}, K_{2}$ and on to $L_{2}$, and continue in this fashion for $k=2,3, \ldots$.

(1) We take $x_{k}^{*}\left(v_{k}\right)=1$ and $x_{k}^{*}(x)=0$ for $y \in\left\{v_{j}, v_{q_{j}}, t_{j}, t_{s_{j}}: j<k\right\}$, and $x_{k} \in \mathcal{Y}$ is sufficiently small; then we can ensure that $I_{k}=L_{k-1}+x_{k}^{*} \otimes x_{k}$ satisfies $I_{k} v_{k}=w_{p_{k}}$ for some $k$.

(2) We take $y_{k}^{*}\left(w_{k}\right)=1$ and $y_{k}(x)=0$ for $x \in\left\{w_{p_{j}}, w_{j}, u_{r_{j}}, u_{j}: j<k\right\} \cup$ $\left\{w_{p_{k}}\right\}$, and $y_{k} \in \mathcal{Y}$ is sufficiently small; then we can ensure that $J_{k}=$ $\left(I_{k}^{-1}+y_{k}^{*} \otimes y_{k}\right)^{-1}$ satisfies $J_{k} v_{q_{k}}=w_{k}$ for some $q_{k}$.

(3) We take $z_{k}^{*}\left(t_{k}\right)=1$ and $z_{k}^{*}(x)=0$ for $x \in \mathcal{Y} \cup\left\{t_{j}, t_{s_{j}}: j<k\right\}$, and $z_{k} \in \mathcal{X}$ is sufficiently small; then we can ensure that $K_{k}=J_{k}+z_{k}^{*} \otimes z_{k}$ satisfies $K_{k} t_{k}=u_{r_{k}}$ for some $k$.

(4) We take $a_{k}^{*}\left(u_{k}\right)=1$ and $a_{k}^{*}(x)=0$ for $x \in \mathcal{Y} \cup\left\{u_{r_{j}}, u_{j}: j<k\right\} \cup\left\{u_{r_{k}}\right\}$, and $a_{k} \in \mathcal{X}$ is sufficiently small; then we can ensure that $L_{k}=\left(K_{k}^{-1}+a_{k}^{*} \otimes a_{k}\right)^{-1}$ satisfies $L_{k} t_{s_{k}}=u_{k}$ for some $s_{k}$.

Provided that the vectors $x_{k}, y_{k}, z_{k}$ and $a_{k}$ are chosen sufficiently small at each stage, these choices ensure that each operator is invertible and maps $\mathcal{Y}$ onto $\mathcal{Y}$, and that the sequence converges in norm to an operator $L$ with the desired properties. 
Finally, to obtain $K$ we proceed as in the proof of Corollary 3.1 using an operator $K=L T$, where $T$ is constructed as in Theorem 2.2. Note that $T V_{2}$ will be independent over $\mathcal{Y}$, since if a vector $u$ satisfies $T u \in \mathcal{Y}$, then $\sum_{n=1}^{\infty} \frac{1}{n^{2}} x_{n}^{*}(u) x_{n} \in \mathcal{Y}$, and so $\sum_{n=1}^{\infty} \frac{1}{n^{2}} w_{n}^{*}(p u) w_{n}=0$ in $\mathcal{X} / \mathcal{Y}$, so that $p u=0$ and $u \in \mathcal{Y}$.

Remark 3.1. (1) It is not sufficient that $V_{1} \cup V_{2}$ and $W_{1} \cup W_{2}$ be independent; if $L \mathcal{Y}=\mathcal{Y}$, then we also require independence of $V_{2}$ and $W_{2}$ over $\mathcal{Y}$ in order to prescribe that $L V_{2}=W_{2}$.

(2) In the situation when we have a chain of subspaces $\mathcal{X}_{1} \subset \mathcal{X}_{2} \subset \mathcal{X}_{3} \subset$ $\ldots \subset \mathcal{X}$, it seems to be possible to make analogous constructions of operators preserving chains of dense independent subsets $V_{1} \subset V_{2} \subset V_{3} \ldots$ and $W_{1} \subset W_{2} \subset W_{3} \ldots$ However, the simplest construction appears to need a complicated diagonal induction, prescribing the image or pre-image in turn of, say,

$$
v_{11}, w_{11}, v_{21}, w_{21}, v_{12}, w_{12}, v_{31}, w_{31}, v_{22}, w_{22}, v_{13}, w_{13}, \ldots
$$

where $v_{i j} \in V_{i}$ and $w_{i j} \in W_{i}$. We omit the details.

\section{ACKNOWLEDGEMENT}

The authors are grateful to an anonymous referee for many helpful comments.

\section{REFERENCES}

[1] S. Grivaux. Construction of operators with prescribed behaviour. Arch. Math. (Basel), 81(3):291-299, 2003. MR2013260 (2004g:47011)

[2] J. Lindenstrauss and L. Tzafriri. Classical Banach spaces. I. Springer-Verlag, Berlin, 1977. Sequence spaces, Ergebnisse der Mathematik und ihrer Grenzgebiete, Vol. 92. MR0500056 $(58: 17766)$

[3] R. I. Ovsepian and A. Pełczyński. On the existence of a fundamental total and bounded biorthogonal sequence in every separable Banach space, and related constructions of uniformly bounded orthonormal systems in $L^{2}$. Studia Math., 54(2):149-159, 1975. MR0394137 $(52: 14942)$

[4] I. Singer. Bases in Banach spaces. II. Springer-Verlag, Berlin-New York, 1981. MR0610799 (82k:46024)

[5] P. Terenzi. Extension of uniformly minimal $M$-basic sequences in Banach spaces. J. London Math. Soc. (2), 27(3):500-506, 1983. MR0697142 (85b:46016)

[6] B. R. Yahaghi. On injective or dense-range operators leaving a given chain of subspaces invariant. Proc. Amer. Math. Soc., 132(4):1059-1066, 2004. MR.2045421 (2005a:47011)

Institut Girard Desargues, UfR de Mathématiques, Université Claude Bernard Lyon 1, 69622 Villeurbanne Cedex, France

E-mail address: chalenda@igd.univ-lyon1.fr

School of Mathematics, University of Leeds, Leeds LS2 9JT, United Kingdom

E-mail address: J.R.Partington@leeds.ac.uk 